\title{
Persistence of local anisotropy of passive scalars in wall-bounded flows
}

\author{
Emmanuel Germaine and Laurent Mydlarski* \\ Department of Mechanical Engineering, McGill University, 817 Sherbrooke Street West, \\ Montréal, Québec H3A 0C3, Canada \\ Luca Cortelezzi \\ Department of Aerospace Science and Technology, Politecnico di Milano, Milano, Italy
}

(Received 30 September 2016; published 17 January 2018)

\begin{abstract}
Local isotropy of passive scalars in fully developed turbulent channel flow is studied by way of direct numerical simulations. We observe a persistent small-scale anisotropy that (i) persists after the flow has undergone substantial mixing and (ii) is independent of the original large-scale anisotropic initial conditions of the scalar field. This latter observation is in sharp contrast with the persistent local anisotropy observed in homogeneous, isotropic turbulence with imposed mean scalar gradients, for which the small-scale anisotropy is directly correlated to the imposed large-scale anisotropy by way of ramp-cliff structures. The anisotropy observed in the present work is linked to the production of $\left\langle\varepsilon_{\theta}\right\rangle$ due to the mean velocity gradient. A major implication of the work is that local isotropy of passive scalar fields may never hold in flows in which mean velocity gradients exist, even after mean scalar gradients have been eliminated by the turbulence.
\end{abstract}

DOI: 10.1103/PhysRevFluids.3.014606

\section{INTRODUCTION}

When a scalar quantity ( $\tilde{\theta}$, i.e., temperature, humidity, chemical-species concentration, etc.) is unevenly distributed within a turbulent flow, the scalar field is stretched and stirred by way of the turbulent mixing process, which serves to deform the scalar gradients. The resulting scalar fluctuations are then smoothed out by the molecular diffusion that principally occurs at the smallest scales of the turbulence.

The predominant theory related to turbulent scalar mixing, Kolmogorov-Obukhov-Corrsin (KOC) theory [1-3], predicts that the small scales should be isotropic and independent of the large scales of a scalar field; the latter being anisotropic in most cases. However, it has been shown that departure from isotropy occurs at the small scales of the scalar field when its large scales are anisotropic, which puts KOC phenomenology into question [4].

Investigations into the local isotropy of the scalar field (and, in particular, violations thereof) have been widely reported in the literature [4-7] and have resulted in significant insight into the origin of such small-scale anisotropy, relating it to the large-scale (ramp-cliff) scalar-field structures that are deemed its cause $[4,8]$. Much of this work, however, has focused on the evolution of (anisotropic) scalar fields within homogeneous and isotropic turbulent hydrodynamic fields [9-12]. However, such a configuration is not representative of typical engineering flows (e.g., duct flows, jets, boundary layers), which are generally inhomogeneous. Moreover, the flows in the aforementioned work are characterized by having persistent mean scalar gradients. Neither the homogeneous, isotropic nature of these flows nor their persistent mean scalar gradients are representative of the substantial classes

*Corresponding author: laurent.mydlarski@mcgill.ca 


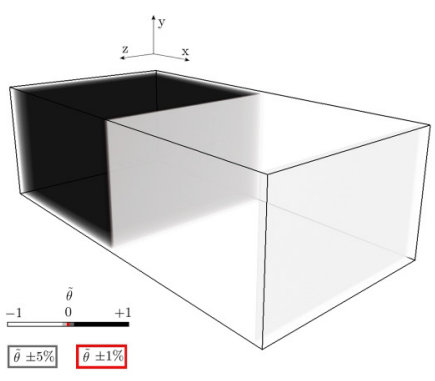

(a)

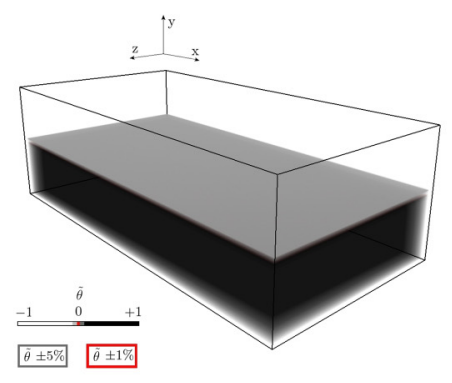

(b)

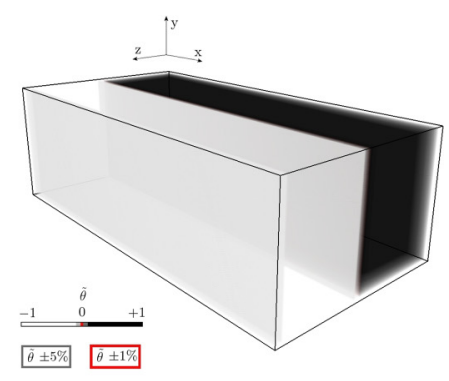

(c)

FIG. 1. Initial configurations of the concentration field: $I C_{x}(\mathrm{a}), I C_{y}(\mathrm{~b}), I C_{z}(\mathrm{c})$.

of flows (e.g., chemical mixers) in which the objective is to eliminate all scalar gradients, with the ultimate goal of producing a homogeneous mixture. Thus, in the present work, we study the evolution of a fundamental wall-bounded flow-a fully developed, high-aspect-ratio, turbulent channel flowfrom (i) an initial condition in which a given scalar gradient is imposed to (ii) a state in which the scalar field has been subjected to substantial mixing by the action of the turbulent fluctuations. The objective of this study is to examine the evolution of the small-scale anisotropy of the passive scalar field from the initial state to later states, which have undergone mixing due to the turbulence.

\section{SIMULATIONS}

Our work is undertaken by means of direct numerical simulation. We begin by simulating (only) the velocity field over a $2 \pi \times 2 \times \pi$ domain and letting it evolve until the flow is fully turbulent, and the statistics of the turbulence are well converged, before proceeding to simulate the scalar field. Once this has occurred, we then "freeze" the velocity field at a given instant of time and consider this instant as the initial time $(t=0)$ for the evolution of the scalar field. We assume that the magnitude of the scalar fluctuations are sufficiently small to consider the scalar as being passive, which renders the problem one-way coupled. Starting from this initial state, we compute the time evolution of the three concentration fields, named $I C_{x}, I C_{y}$, and $I C_{z}$, shown in Figs. 1(a)-1(c), respectively. These fields consist of two equal volumes of uniform concentrations, $\tilde{\theta}=-1$ (white) and 1 (black), separated by two interfaces (due to the periodic boundary conditions in $x$ and $z$ ) of total area $4 \pi(=2 \times 2 \times \pi)$ for $I C_{x}$ and $8 \pi(=2 \times 2 \times 2 \pi)$ for $I C_{z}$ and a single interface of area $2 \pi^{2}(=2 \pi \times \pi)$ for $I C_{y}$, and all fields have zero mean concentration (see Fig. 1). The motivation for studying these three initial conditions is to investigate the evolution of the small-scale scalar field subjected to different initial conditions. Note that each initial concentration field is subject to the action of an identical turbulent velocity field. Given that this velocity field is inhomogeneous (unlike the previously mentioned works studying the small-scale statistics of the scalar field subjected to homogeneous, isotropic turbulent velocity fields), changing the orientation of the initial scalar field (with respect to the velocity field) will now produce different scalar fields.

We numerically solve the advection-diffusion equation

$$
\frac{\partial \tilde{\theta}}{\partial t}+\tilde{u}_{j} \frac{\partial \tilde{\theta}}{\partial x_{j}}=\alpha \frac{\partial^{2} \tilde{\theta}}{\partial x_{j} \partial x_{j}},
$$

where $\tilde{\theta}$ represents the instantaneous scalar (temperature) field, $\tilde{u}_{j}, j=1,2,3$ are the components of the instantaneous velocity vector field, $\alpha$ is the thermal diffusivity of the fluid (air), and repeated indices imply Einstein's summation convention. In our subsequent discussion, we will decompose the instantaneous scalar and velocity fields ( $\tilde{\theta}$ and $\tilde{u}_{j}$, respectively), into their respective mean ( $T$ and $U_{j}$ ) and fluctuating $\left(\theta\right.$ and $\left.u_{j}\right)$ quantities. (We note that the hydrodynamic field herein is statistically steady and statistically one-dimensional, whereas the scalar field is unsteady and three-dimensional. 


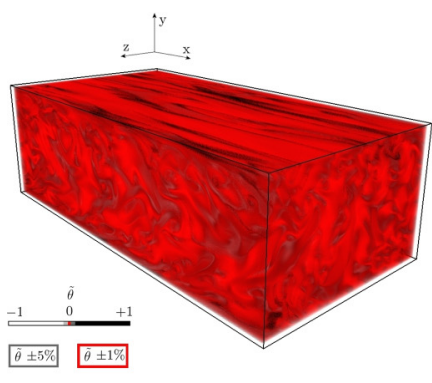

(a)

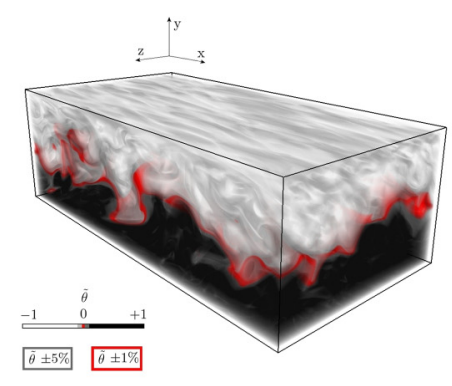

(b)

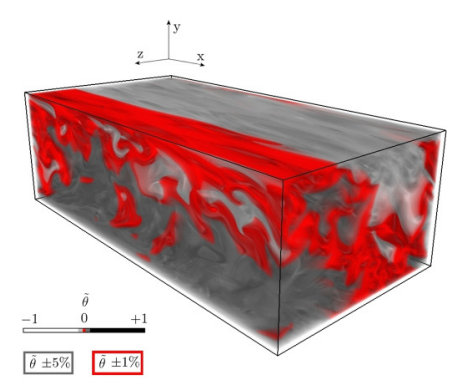

(c)

FIG. 2. Concentration distributions generated at $\tau=7$ by the action of the same fully turbulent channel flow on the initial conditions: $I C_{x}(\mathrm{a}), I C_{y}(\mathrm{~b})$, and $I C_{z}(\mathrm{c})$.

Thus, suitable definitions of averages of fluctuating quantities must be chosen accordingly. The selection of appropriate averages is crucial to the analysis of the results, and will be addressed in more detail.) Moreover, the assumption that the scalar field is passive allows us to solve Eq. (1) by first precomputing, for the entire duration of the numerical experiment, the fully turbulent velocity vector field $\tilde{u}_{j}$. For this task, we use a spectral method in space (Fourier $\times$ Chebyshev $\times$ Fourier) and a third-order Runge-Kutta integrator in time, with no-penetration/no-slip boundary conditions imposed at the walls of the channel ( $y=0$ and $y=2 h$, where $h$ is the half-height of the channel), and periodic boundary conditions in the streamwise, $x$, and spanwise, $z$, directions implemented in the spectral code entitled CHANNELFLOW $[13,14]$. The resulting fully developed turbulent velocity field is statistically one-dimensional, such that $U=U(y)$ only. It is precomputed at $\operatorname{Re}_{\tau}=190$ (where $\operatorname{Re}_{\tau}=u_{\tau} h / v, u_{\tau} \equiv \sqrt{\nu \times \partial U /\left.\partial y\right|_{y=0}}$, and $v$ is the kinematic viscosity of the fluid) over a domain $2 \pi \times 2 \times \pi$ with a resolution of $256 \times 193 \times 192$. The velocity field (only) is the same as that used in Ref. [15].

We then compute the time evolution of the scalar field by solving the advection-diffusion equation (1) using a third-order flux integral method combined with a second-order semi-Lagrangian time integrator [16] with adiabatic boundary conditions at the walls of the channel and periodic boundary conditions in the streamwise, $x$, and spanwise, $z$, directions. The time evolution of the scalar field is computed over the same $2 \pi \times 2 \times \pi$ domain with a resolution of $514 \times 195 \times 194$ to resolve both the large and small scales of the turbulent scalar field. In the present work, time will be nondimensionalized by an eddy turnover time such that $\tau \equiv u_{\tau} t / h$. The Péclet number of the flow is $\mathrm{Pe}=2550$ (where $\mathrm{Pe} \equiv U_{y / h=1} h / \alpha$, where $U_{y / h=1}$ is the mean longitudinal centreline velocity of the fluid in the channel) and the Prandtl number is 0.71 , such that $\operatorname{Re}\left(\equiv U_{y / h=1} h / v\right)=3590$.

\section{RESULTS}

Figures 2(a)-2(c) show the resulting concentration distributions at $\tau=7$ by the action of the same fully turbulent channel flow on the highly anisotropic initial conditions $I C_{x}, I C_{y}$, and $I C_{z}$, respectively, shown in Fig. 1. Before considering the small-scale statistics of the passive scalar field, it is interesting to note how differently the initial concentration distributions are mixed by the same fully turbulent channel flow, or, in other words, how turbulent mixing over a moderately long time (seven eddy turnover times) depends so strongly on the geometry of the initial concentration field. The initial concentration field $I C_{x}$ [Fig. 2(a)] appears to be quite well mixed at $\tau=7$ (being mostly red, i.e., mixed to within $1 \%$ of the mean), while the field $I C_{y}$ [Fig. 2(b)] shows two very poorly mixed layers of "black" and "white" fluids. The initial concentration field $I C_{z}$ falls in between, being moderately mixed [Fig. 2(c)].

A critical term in the evolution of the scalar fields is the rate of destruction of the scalar variance [the so-called scalar dissipation rate, $\left\langle\varepsilon_{\theta}\right\rangle \equiv \alpha\left\langle\left(\partial \theta / \partial x_{i}\right)^{2}\right\rangle$ ], although the latter name is somewhat of 


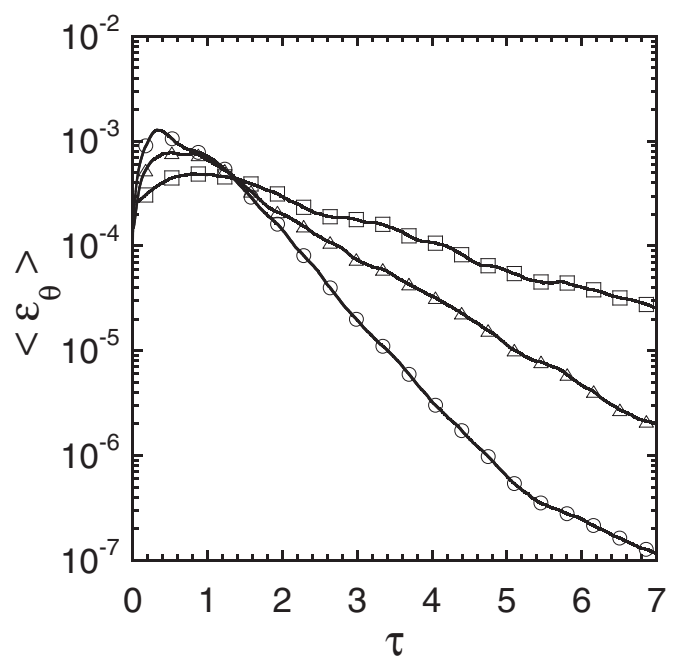

FIG. 3. Time-evolution of the scalar dissipation rate for the three initial conditions: $I C_{x}$ (circles), $I C_{y}$ (squares), $I C_{z}$ (triangles).

a misnomer, as it is not the scalar itself that is dissipated, but its fluctuations/variance. $\left\langle\varepsilon_{\theta}\right\rangle$ is the only term in the scalar variance budget that is nonzero in every turbulent flow. Consequently, $\left\langle\varepsilon_{\theta}\right\rangle$ is omnipresent and of critical importance to the description of turbulent scalar fields, and therefore plays a key role in KOC theory. Moreover, and of particular interest to the focus of the present work, $\left\langle\varepsilon_{\theta}\right\rangle$ is a quantity whose primary contributions derive from the smallest scales of the scalar field. Also note that the angular brackets used in the definition of $\left\langle\varepsilon_{\theta}\right\rangle$ will denote volume averaging herein. This choice of average, as alluded to earlier, is a consequence of the unsteady, three-dimensional nature of the scalar field, and its implication to our subsequent results and analysis will be further discussed.

Figure 3 shows the evolution of $\left\langle\varepsilon_{\theta}\right\rangle$ as a function of the eddy turnover time, $\tau \equiv u_{\tau} t / h$, for the three initial conditions: $I C_{x}$ (circles), $I C_{y}$ (squares), and $I C_{z}$ (triangles). The scalar dissipation rate increases at early times because the turbulent velocity field stretches and stirs the interface between black and white fluids, effectively distributing small-scale gradients. This process is most efficient for the initial concentration field $I C_{x}$, which presents the highest peak, because the interfaces between black and white fluids are anchored to the walls of the channel (by the no-slip boundary condition) and, therefore, strongly stretched (especially near the walls) and efficiently stirred by the turbulent flow (especially by the near-wall turbulence). The production of $\left\langle\varepsilon_{\theta}\right\rangle$ at early times is less intense for the $I C_{z}$ initial condition. Although the two interfaces are anchored to the walls of the channel by the no-slip boundary condition, as in the case $I C_{x}$, they are aligned with the streamwise direction and, therefore, are only stretched and stirred by the spanwise turbulent flow, which is much weaker than the streamwise one. The least efficient mixing occurs for the $I C_{y}$ case, which exhibits the lowest peak, because the entire interface between black and white fluid, which initially coincides with the mid-plane of the channel, is advected by the turbulent flow at the center of the channel and, therefore, weakly stretched and stirred by it.

In all cases, after about one eddy turnover time, the scalar dissipation rate starts decreasing steadily at a constant rate that clearly depends on the geometry of the initial conditions. The scalar dissipation rate decreases faster for the initial condition $I C_{x}$ and substantially slower for the case $I C_{y}$. We speculate that the change in slope of the curve for the $I C_{x}$ case, at around $\tau=5.5$, is due to the transition from an advective-diffusive phase to a purely diffusive one. The evolution of the scalar dissipation rate in the case $I C_{z}$ falls in between the other two cases. Note that the time evolution of the scalar dissipation rate (Fig. 3) and the evolution of the concentration field (Fig. 2) are intuitively 


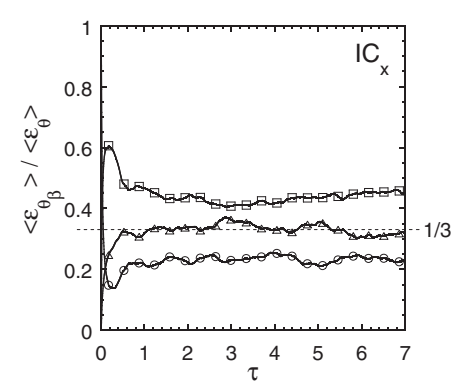

(a)

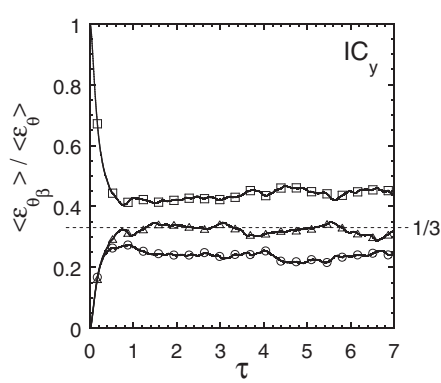

(b)

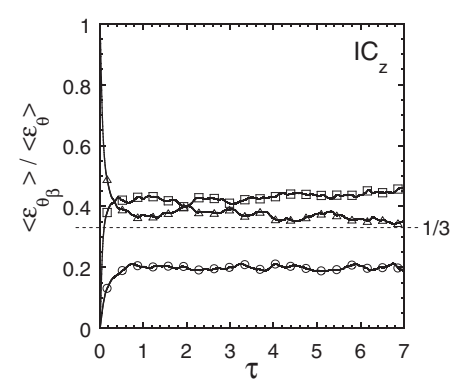

(c)

FIG. 4. Time-evolution of the fractional components of the scalar dissipation rate, $\left\langle\varepsilon_{\theta_{x}}\right\rangle /\left\langle\varepsilon_{\theta}\right\rangle$ (circles), $\left\langle\varepsilon_{\theta_{y}}\right\rangle /\left\langle\varepsilon_{\theta}\right\rangle$ (squares) and $\left\langle\varepsilon_{\theta_{z}}\right\rangle /\left\langle\varepsilon_{\theta}\right\rangle$ (triangles) for the three initial conditions: $I C_{x}$ (a), $I C_{y}$ (b), and $I C_{z}$ (c).

coherent, the higher the destruction of the scalar variance being associated with better mixing of the scalar field.

From Fig. 3, it is clear that the evolution of $\left\langle\varepsilon_{\theta}\right\rangle$ (a small-scale quantity) is sensitive to the scalar-field initial conditions (a large-scale condition). As a result, it is reasonable to extrapolate that individual components of the scalar dissipation rate $\left(\left\langle\varepsilon_{\theta_{x}}\right\rangle \equiv \alpha\left\langle(\partial \theta / \partial x)^{2}\right\rangle,\left\langle\varepsilon_{\theta_{y}}\right\rangle \equiv \alpha\left\langle(\partial \theta / \partial y)^{2}\right\rangle\right.$, and $\left.\left\langle\varepsilon_{\theta_{z}}\right\rangle \equiv \alpha\left\langle(\partial \theta / \partial z)^{2}\right\rangle\right)$ might also be affected by the large-scale anisotropic scalar-field initial conditions.

To investigate this possibility, we plot in Fig. 4 the fractional components of the scalar dissipation rate as a function of time to quantify the evolution of the small-scale anisotropy of the passive scalar field from the (anisotropic) initial state shown in Fig. 1 to the final states shown in Fig. 2. The results shown in Fig. 4 are somewhat surprising. One would expect the level of anisotropy to be different from case to case, with the small scales of the scalar field associated with the best-mixed initial condition $\left(I C_{x}\right)$ to be closest to their isotropic value $(1 / 3)$ and those associated with the least-mixed case $\left(I C_{y}\right)$ to be farthest from isotropy.

This intuitive expectation is contradicted by Fig. 4. At $\tau=0$, in all cases, due to the high anisotropy of the initial conditions, two fractional components are zero and the remaining equals one, where the nonzero component is $\left\langle\varepsilon_{\theta_{x}}\right\rangle /\left\langle\varepsilon_{\theta}\right\rangle$ in the $I C_{x}$ case, $\left\langle\varepsilon_{\theta_{y}}\right\rangle /\left\langle\varepsilon_{\theta}\right\rangle$ in the $I C_{y}$ case, and $\left\langle\varepsilon_{\theta_{z}}\right\rangle /\left\langle\varepsilon_{\theta}\right\rangle$ in the $I C_{z}$ case. After a short transient of about half an eddy turnover time, in all cases, all the fractional components reach a reasonably stable value, with only $\left\langle\varepsilon_{\theta_{z}}\right\rangle /\left\langle\varepsilon_{\theta}\right\rangle$ being close to or approaching the theoretical value of $1 / 3$. The other two fractional components, $\left\langle\varepsilon_{\theta_{x}}\right\rangle /\left\langle\varepsilon_{\theta}\right\rangle$ and $\left\langle\varepsilon_{\theta_{y}}\right\rangle /\left\langle\varepsilon_{\theta}\right\rangle$, are substantially and consistently below and above the value $1 / 3$, respectively, in all cases. Therefore, Fig. 4 strongly indicates that in the case of fully turbulent channel flows there is a persistent anisotropy at small scales and the relative magnitude of the fractional components of the scalar dissipation rate are independent of the initial conditions of the scalar field.

To analyze the lack of return to isotropy presented above, one's first instinct is to study the evolution equation of the scalar dissipation rate:

$$
\begin{aligned}
\frac{\partial\left\langle\varepsilon_{\theta}\right\rangle}{\partial t}+U_{j} \frac{\partial\left\langle\varepsilon_{\theta}\right\rangle}{\partial x_{j}}= & \overbrace{-2 \alpha \frac{\partial U_{j}}{\partial x_{i}}\left\langle\frac{\partial \theta}{\partial x_{i}} \frac{\partial \theta}{\partial x_{j}}\right\rangle}^{\mathcal{P}}-2 \alpha \frac{\partial T}{\partial x_{j}}\left\langle\frac{\partial u_{j}}{\partial x_{i}} \frac{\partial \theta}{\partial x_{i}}\right\rangle-2 \alpha\left\langle u_{j} \frac{\partial \theta}{\partial x_{i}}\right\rangle \frac{\partial^{2} T}{\partial x_{i} \partial x_{j}} \\
& -2 \alpha\left\langle\frac{\partial u_{j}}{\partial x_{i}} \frac{\partial \theta}{\partial x_{i}} \frac{\partial \theta}{\partial x_{j}}\right\rangle+\frac{\partial}{\partial x_{j}}\left(\alpha \frac{\partial\left\langle\varepsilon_{\theta}\right\rangle}{\partial x_{j}}-\left\langle u_{j} \varepsilon_{\theta}\right\rangle\right)-\underbrace{2 \alpha^{2}\left\langle\frac{\partial^{2} \theta}{\partial x_{i} \partial x_{j}} \frac{\partial^{2} \theta}{\partial x_{i} \partial x_{j}}\right\rangle}_{\Gamma} .
\end{aligned}
$$




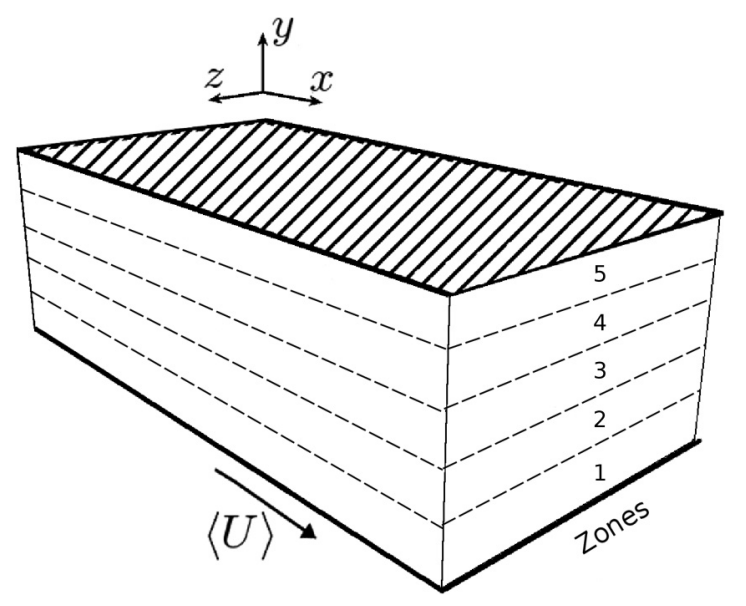

FIG. 5. Schematic of the subdivision of the computational domain to investigate small scale anisotropy.

In the limit of large Reynolds and Péclet numbers, it is hypothesized [17,18] that the above equation simplifies to a balance between the production of $\left\langle\varepsilon_{\theta}\right\rangle$, due to intensification of the scalar gradients by the turbulent strain rate $\left(2 \alpha\left\langle\frac{\partial u_{j}}{\partial x_{i}} \frac{\partial \theta}{\partial x_{i}} \frac{\partial \theta}{\partial x_{j}}\right\rangle\right)$, and the destruction of $\left\langle\varepsilon_{\theta}\right\rangle$ by molecular processes $\left(\Gamma=2 \alpha^{2}\left\langle\frac{\partial^{2} \theta}{\partial x_{i} \partial x_{j}} \frac{\partial^{2} \theta}{\partial x_{i} \partial x_{j}}\right\rangle\right)$. However, other terms may be relevant-at least at the finite Reynolds and Péclet numbers of the present simulations. One hypothesis is that the production of $\left\langle\varepsilon_{\theta}\right\rangle$ attributed to mean velocity gradients [the term labeled $\mathcal{P}=\mathcal{P}_{y}=-2 \alpha \partial U / \partial y\langle(\partial \theta / \partial y)(\partial \theta / \partial x)\rangle$ in Eq. (2)] may not be negligible. Moreover, because there is only one velocity gradient in the flow under consideration, $\mathcal{P}_{y}$ is the only nonzero component of $\mathcal{P}\left(\mathcal{P}_{x}=\mathcal{P}_{z}=0\right)$. This hypothesis is consistent with the results of Fig. 4, which indicate that $\left\langle\varepsilon_{\theta_{y}}\right\rangle$ is largest of the three components of $\left\langle\varepsilon_{\theta}\right\rangle$.

To confirm this hypothesis, it would be of benefit to compare $\mathcal{P}$ with the leading-order term in Eq. (2): $2 \alpha\left\langle\frac{\partial u_{j}}{\partial x_{i}} \frac{\partial \theta}{\partial x_{i}} \frac{\partial \theta}{\partial x_{j}}\right\rangle$. However, calculation of the individual terms in Eq. (2) is not as evident as it may initially appear for the following reasons. First, in the flow herein, the scalar field is unsteady and three-dimensional, whereas the hydrodynamic field is statistically steady and (statistically) onedimensional. This inconsistency in the underlying statistical properties of the flow precludes the use of a consistent definition of an average to use for the two fields. Specifically, the unsteady, threedimensional nature of the scalar field precludes the use of either time or (one- or two-dimensional) spatial averages to calculate statistics of the scalar field at a given point (or line or plane) in the flow-hence our choice of volume averages used in Figs. 3 and 4. And although the hydrodynamic field naturally lends itself to the use of averages in time and/or averages in space evaluated over the $x$ and $z$ directions (in which the hydrodynamic field is homogeneous), applying the same average to the scalar field is not justifiable, as just noted. Conversely, use of a volume average to describe the hydrodynamic field will not capture relevant physical phenomena, such as the mean velocity gradient. Thus, no suitable choice of an average exists. Second, the different statistical nature of the two fields not only causes problems with respect to the averaging of the quantities in Eq. (2), but it also causes inconsistencies with respect to the definitions of the fluctuating quantities $\left(\theta\right.$ and $\left.u_{j}\right)$, as these can only be defined relative to their respective average values ( $T$ being the average of $\tilde{\theta}$, and $U_{j}$ being the average of $\tilde{u}_{j}$ ).

Given this issue, we have chosen an alternate method to investigate the source of the sustained anisotropy at small scales. As depicted in Fig. 5, we subdivide the computational domain into five layered regions (parallel to the walls) of equal height: Zone 1, the near-wall region, where $0<y^{+}<75$; Zone 2, the intermediate region, where $75<y^{+}<151$; Zone 3, the central-channel region, where $151<y^{+}<228$; and Zones 4 and 5 being the mirror images of Zones 2 and 1 , respectively, with respect to the center plane of the channel. We recompute the fractional components 


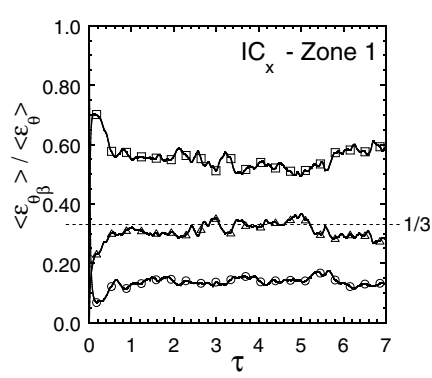

(a)

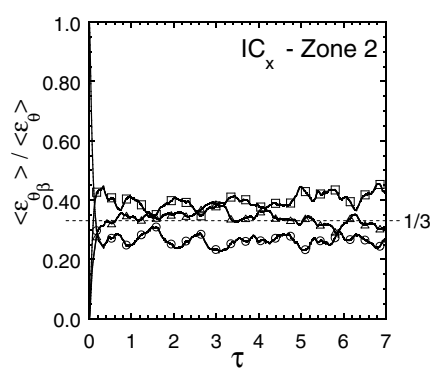

(d)

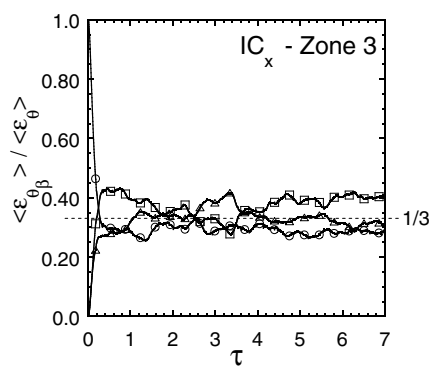

(g)

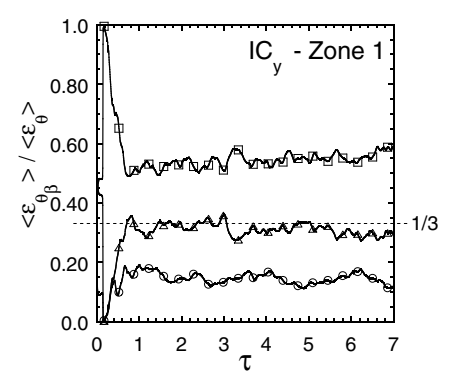

(b)

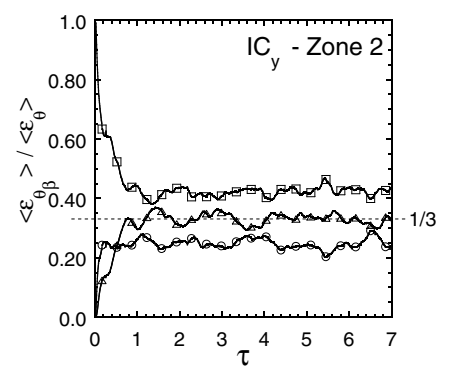

(e)

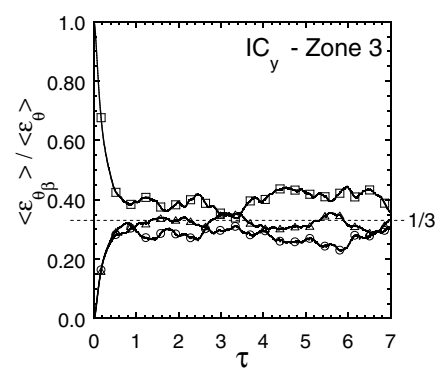

(h)

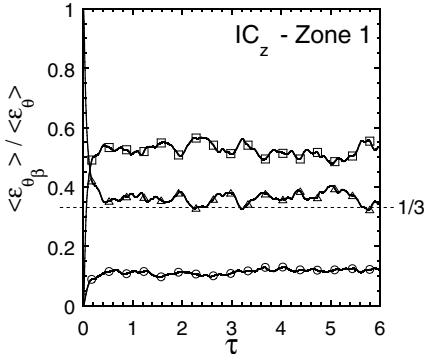

(c)

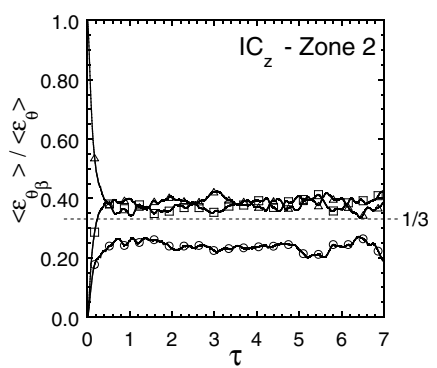

(f)

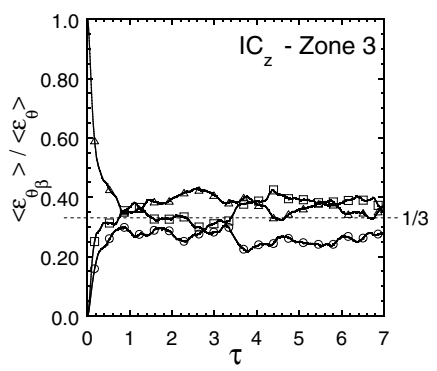

(i)

FIG. 6. Time evolution of the fractional components of the scalar dissipation rate, $\left\langle\varepsilon_{\theta_{x}}\right\rangle /\left\langle\varepsilon_{\theta}\right\rangle$ (circles), $\left\langle\varepsilon_{\theta_{y}}\right\rangle /\left\langle\varepsilon_{\theta}\right\rangle$ (squares), and $\left\langle\varepsilon_{\theta_{z}}\right\rangle /\left\langle\varepsilon_{\theta}\right\rangle$ (triangles) in Zone 1 (a), (b), (c), Zone 2 (d), (e), (f), and Zone 3 (g), (h), (i) for the three initial conditions $I C_{x}(\mathrm{a}),(\mathrm{d}),(\mathrm{g}), I C_{y}(\mathrm{~b}),(\mathrm{e}),(\mathrm{h})$ and $I C_{z}(\mathrm{c}),(\mathrm{f}),(\mathrm{i})$.

of turbulent dissipation rate by averaging them, in this case, over the volume of each single zone, instead of averaging over the entire volume of the computational domain. Note that at time $\tau=0$, for the case $I C_{y}$, the Zones 1 and 2 are filled with black fluid only. The interface is completely contained in Zone 3, where half of the fluid is black and half white. Because of this unique situation, the values of $\left\langle\varepsilon_{\theta_{x}}\right\rangle,\left\langle\varepsilon_{\theta_{y}}\right\rangle,\left\langle\varepsilon_{\theta_{z}}\right\rangle$, and $\left\langle\varepsilon_{\theta}\right\rangle$ are all simultaneously zero at $\tau=0$ in Zones 1 and 2 and, consequently, the fractional components are undetermined. We resolved this problem by forcing the fractional components to be zero until $\left\langle\varepsilon_{\theta}\right\rangle$ (averaged over the volume of a single zone) has reached a small but meaningful value (i.e., a value a few orders of magnitudes above machine precision).

Figure 6 shows the time-evolution of the fractional components of the scalar dissipation rate, $\left\langle\varepsilon_{\theta_{x}}\right\rangle /\left\langle\varepsilon_{\theta}\right\rangle$ (circles), $\left\langle\varepsilon_{\theta_{y}}\right\rangle /\left\langle\varepsilon_{\theta}\right\rangle$ (squares), and $\left\langle\varepsilon_{\theta_{z}}\right\rangle /\left\langle\varepsilon_{\theta}\right\rangle$ (triangles) in Zone 1 (a, b, c), Zone 2 (d, e, f), and Zone $3(\mathrm{~g}, \mathrm{~h}, \mathrm{i})$ for the three initial conditions: $I C_{x}(\mathrm{a}, \mathrm{d}, \mathrm{g}), I C_{y}(\mathrm{~b}, \mathrm{e}, \mathrm{h})$, and $I C_{z}(\mathrm{c}, \mathrm{f}, \mathrm{i})$. The plots of the fractional components in each zone show that a persistent small-scale anisotropy (i) is clearly localized near the walls of the channel (i.e., in Zone 1), and (ii) appears to be independent of the initial condition of the scalar field. Moreover, this small-scale anisotropy disappears in the center of the channel (i.e., Zone 3), where $\partial U / \partial y \approx 0$ and where the turbulence appears to be isotropic 
at small scales. Thus, the small-scale anisotropy of the scalar field appears to be sustained by the gradients in the mean velocity of the hydrodynamic field.

\section{CONCLUSIONS}

In conclusion, we have demonstrated that in a wall-bounded flow with mean velocity gradients there exists a persistent small-scale anisotropy of a passive scalar field. Moreover, this small-scale anisotropy is independent of the scalar field initial conditions, even when the total scalar dissipation rate, $\left\langle\varepsilon_{\theta}\right\rangle$, continues to exhibit a behavior that can be correlated with the same scalar field initial conditions. We argue that this small-scale anisotropy results from the production of $\left\langle\varepsilon_{\theta}\right\rangle$ arising from the mean velocity gradient. Although this observation is not inconsistent with the evolution equation for $\left\langle\varepsilon_{\theta}\right\rangle$ (at least at noninfinite Reynolds and Péclet numbers), it may indeed contradict KolmogorovObukhov-Corrsin (KOC) theory [1-3], as it may imply that any flows with constant mean velocity gradients will invariably exhibit persistent small-scale anisotropy of the scalar field, even if the large scales of the scalar field are well mixed. This mechanism is distinctly different from the one that results in persistence of local anisotropy for scalar fields that are observed in homogeneous, isotropic turbulence with mean scalar gradients. And although there remains a possibility that the anisotropy observed herein might disappear as the Reynolds and Péclet numbers tend to infinity, we remark that (i) the anisotropy exhibits no tendency whatsoever to reduce in magnitude as the scalar field homogenizes, thus potentially indicating that it could also be insensitive to the increased mixing and separation of scales associated with higher Reynolds and Péclet numbers, and (ii) the passive scalar field has already shown that it exhibits nondecaying, small-scale anisotropies in homogeneous, isotropic turbulence with mean scalar gradients [4,9-11]. In any case, the results herein further confirm the unique nature and behavior of passive scalar fields, especially with respect to Kolmogorov theory. Last, future work exploring the persistence of the observed small-scale anisotropy of a passive scalar field at higher Reynolds and Péclet numbers - whether it be experimentally or numericallywould clearly be of interest. However, the current results nevertheless indicate that small-scale anisotropy will be present at finite Reynolds and Péclet numbers, and that application of the notions of Kolmogorov without careful consideration of their range of applicability may lead to erroneous conclusions in the vast array of flows that cannot be reasonably modeled as being of infinite Reynolds number.

\section{ACKNOWLEDGMENTS}

The authors would like to thank the anonymous reviewers for their constructive comments. Moreover, funding for this work was generously provided by the Natural Sciences and Engineering Research Council of Canada under Contracts No. RGPIN217169 and No. RGPIN217184.

[1] A. Kolmogorov, The local structure of turbulence in incompressible viscous fluid for very large Reynolds numbers, Dokl. Akad. Nauk SSSR 30, 301 (1941).

[2] A. M. Obukhov, Structure of the temperature field in a turbulent current, Izv. Akad. Nauk. SSSR, Ser. Geograf. Geofiz 13, 58 (1949).

[3] S. Corrsin, On the spectrum of isotropic temperature fluctuations in an isotropic turbulence, J. Appl. Phys. 22, 469 (1951).

[4] Z. Warhaft, Passive scalars in turbulent flows, Annu. Rev. Fluid Mech. 32, 203 (2000).

[5] R. A. Antonia and C. W. Van Atta, Structure functions of temperature fluctuations in turbulent shear flows, J. Fluid Mech. 84, 561 (1978).

[6] K. R. Sreenivasan, On local isotropy of passive scalars in turbulent shear flows, Proc. R. Soc. London, Ser. A 434, 165 (1991). 
[7] B. I. Shraiman and E. D. Siggia, Scalar turbulence, Nature (London) 405, 639 (2000).

[8] R. A. Antonia, A. J. Chambers, D. Britz, and L. W. B. Browne, Organized structures in a turbulent plane jet: Topology and contribution to momentum and heat-transport, J. Fluid Mech. 172, 211 (1986).

[9] M. Holzer and E. D. Siggia, Turbulent mixing of a passive scalar, Phys. Fluids 6, 1820 (1994).

[10] C. Tong and Z. Warhaft, On passive scalar derivative statistics in grid turbulence, Phys. Fluids 6, 2165 (1994).

[11] L. Mydlarski and Z. Warhaft, Passive scalar statistics in high-Péclet-number grid turbulence, J. Fluid Mech. 358, 135 (1998).

[12] W. J. T. Bos, On the anisotropy of the turbulent passive scalar in the presence of a mean scalar gradient, J. Fluid Mech. 744, 38 (2014).

[13] J. F. Gibson, J. Halcrow, and P. Cvitanović, Visualizing the geometry of state space in plane Couette flow, J. Fluid Mech. 611, 107 (2008).

[14] J. F. Gibson, Channelflow: A spectral Navier-Stokes simulator in C++, http://channelflow.org/, University of New Hampshire, 2010.

[15] E. Germaine, L. Mydlarski, and L. Cortelezzi, Evolution of the scalar dissipation rate downstream of a concentrated line source in turbulent channel flow, J. Fluid Mech. 749, 227 (2014).

[16] E. Germaine, L. Mydlarski, and L. Cortelezzi, 3DFLUX: A high-order fully three-dimensional flux integral solver for the scalar transport equation, J. Comput. Phys. 240, 121 (2013).

[17] S. Corrsin, Remarks on turbulent heat transfer: An account of some features of the phenomenon in fully turbulent regions, in Proceedings of the First Iowa Thermodynamics Symposium (University of Iowa, Iowa City, 1953), p. 5.

[18] H. Tennekes and J. L. Lumley, A First Course in Turbulence (MIT Press, Cambridge, MA, 1972). 